\title{
Endovascular Treatment of Internal Carotid Artery Bifurcation Aneurysms: A Single-Center Experience and a Systematic Review and Meta-Analysis
}

\author{
S.F. Morales-Valero, W. Brinjikji, M.H. Murad, J.T. Wald, and G. Lanzino
}

\begin{abstract}
BACKGROUND AND PURPOSE: Endovascular coiling of internal carotid artery bifurcation aneurysms can be challenging due to unfavorable morphologic features. With improvements in endovascular techniques, several series have detailed the results and complications of endovascular treatment of aneurysms at this location. We performed a systematic review and meta-analysis of published series on the endovascular treatment of ICA bifurcation aneurysms, including a tertiary referral center experience.
\end{abstract}

MATERIALS AND METHODS: We performed a comprehensive literature search for reports on contemporary endovascular treatment of ICA bifurcation aneurysms from 2000 to 2013, and we reviewed our experience. We extracted information regarding periprocedural complications, procedure-related morbidity and mortality, immediate angiographic outcome, long-term clinical and angiographic outcome, and retreatment rate. Event rates were pooled across studies by using random-effects meta-analysis.

RESULTS: Including our series of 37 patients, 6 studies with 158 patients were analyzed. Approximately $60 \%$ of the aneurysms presented as unruptured; $88.0 \%(95 \% \mathrm{Cl}, 68.0 \%-96.0 \%)$ of aneurysms showed complete or near-complete occlusion at immediate postoperative angiography compared with $82.0 \%(95 \% \mathrm{Cl}, 73.0 \%-88.0 \%)$ at last follow-up. The procedure-related morbidity and mortality were $3.0 \%(95 \%$ $\mathrm{Cl}, 1.0 \%-7.0 \%)$ and $3.0 \%(95 \% \mathrm{Cl}, 1.0 \%-8.0 \%)$, respectively. The retreatment rate was $14.0 \%(95 \% \mathrm{Cl}, 8.0 \%-25.0 \%)$. Good neurologic outcome was achieved in $93.0 \%(95 \% \mathrm{Cl}, 86.0 \%-97.0 \%)$ of patients.

CONCLUSIONS: Endovascular treatment of ICA bifurcation aneurysms is feasible and effective and is associated with high immediate angiographic occlusion rates. However, retreatment rates and procedure-related morbidity and mortality are non-negligible.

I

nternal carotid artery bifurcation aneurysms represent between

$2.4 \%$ and $4 \%$ of all intracranial aneurysms. ${ }^{1-4}$ The presence of multiple perforators in this area along with the angle of origin (often skewed toward the MCA or the anterior cerebral artery primarily) can make treatment challenging. ${ }^{3}$ Additionally, the increased hemodynamic stress at this level translates into a higher rate of recurrence compared with aneurysms in other locations. ${ }^{4,5}$ Several studies have focused on the surgical management of ICA bifurcation aneurysms. ${ }^{2,3,6-10}$ However, to our knowledge, there is limited evidence regarding their treatment by using endovascular techniques. To better understand the safety and efficacy of endovascular treatment for ICA bifurcation aneurysms, we report

Received February 6, 2014; accepted after revision March 31.

From the Departments of Neurologic Surgery (S.F.M.-V., G.L.) and Radiology (W.B., J.T.W., G.L.), and Center for Science of Healthcare Delivery (M.H.M.), Mayo Clinic, Rochester, Minnesota.

Please address correspondence to Giuseppe Lanzino, MD, Department of Neurologic Surgery, Mayo Clinic, 200 SW First St, Rochester, MN 55905; e-mail: lanzino. giuseppe@mayo.edu

http://dx.doi.org/10.3174/ajnr.A3992 both our own experience and the results of a meta-analysis of the literature.

\section{MATERIALS AND METHODS}

\section{Patient and Aneurysm Characteristics}

After institutional review board approval, we performed a retrospective analysis of a prospectively collected data base of patients treated for intracranial aneurysms at our institution (Mayo Clinic, Rochester, Minnesota). All consecutive patients who underwent endovascular treatment of aneurysms located at the ICA bifurcation between February 1999 and November 2013 were identified. All patients provided approval for the use of their medical records for retrospective analysis. The following information was collected for each patient: demographic data, aneurysm characteristics, clinical presentation, and type of treatment. Among the aneurysm characteristics, size (maximum dimension as measured by $3 \mathrm{D}$ digital subtraction angiography) and rupture status were obtained. The patients presenting with subarachnoid hemorrhage were classified by using the Hunt and Hess scale score, and clinical outcomes were assessed by using the modified Rankin Scale. 


\section{Outcomes}

Outcomes studied included periprocedural complications $(<30$ days), immediate angiographic outcome, clinical and angiographic outcomes at last follow-up, and retreatment. Studied periprocedural complications (within 30 days of treatment) included intraoperative rupture, thromboembolic events, parent artery and branch occlusion, stroke, vasospasm, and rebleeding.

The degree of aneurysm occlusion was stratified into 3 levels, according to the scale of Raymond et al (complete, near-complete, and incomplete occlusion). ${ }^{11}$ This parameter was established immediately after the procedure and at last follow-up by the operator after reviewing the postoperative 3D-DSA images. Additionally, an experienced neuroradiologist not involved in the patient treatment reviewed the imaging and estimated the percentage of occlusion after the initial embolization and at last follow-up.

\section{Literature Review}

A comprehensive literature search of the data bases PubMed, Ovid MEDLINE, and Ovid EMBASE was designed and conducted by an experienced librarian with input from the authors. The key words "internal carotid artery," "intracranial aneurysm," "endovascular therapy," "coil," "bifurcation," and "terminus" were used in both "AND" and "OR" combinations. The search was limited to articles published from 2000 to November 2013 in the English language only. All studies reporting patients with ICA bifurcation aneurysms treated with endovascular therapy were selected. The inclusion criteria were the following: a series of $>10$ patients, with available data on periprocedural complications and clinical and angiographic follow-up. Two reviewers (S.F.M.-V. and W.B.) independently selected the included studies. For each study, we independently extracted the following information: rupture status, immediate and delayed angiographic occlusion rates, procedure-related morbidity and mortality, periprocedural stroke resulting in morbidity or mortality, intraoperative rupture rates, retreatment rates, and the rate of good neurologic outcome at last follow-up. For patients with ruptured aneurysms, we studied the rate of mortality attributed to complications of SAH, vasospasm rates, and rates of rerupture after coiling.

\section{Statistical Analysis}

We assessed the cumulative incidence (event rate) and 95\% confidence interval for each outcome. Cumulative outcomes (ruptured and unruptured) were also calculated for all results. Perioperative complication rates and clinical outcomes were calculated separately for ruptured and unruptured aneurysms. For metaanalysis, event rates were pooled across studies by using a random-effects model. ${ }^{12}$ Heterogeneity across studies was evaluated by using the $\mathrm{I}^{2}$ statistic. ${ }^{13}$ All statistical analyses were performed by using the meta-analysis software package Comprehensive Meta-Analysis (www.meta-analysis.com).

\section{RESULTS}

\section{Institutional Series}

Patient and Aneurysm Characteristics. Thirty-six consecutive patients (mean age, 50.7 years) with 37 ICA bifurcation aneurysms were included in the current series (22 female patients,
$61.1 \%)$. The mean aneurysm size was $8.8 \pm 5.1 \mathrm{~mm}$. Specifically, there were 12 small aneurysms ( $\leq 5 \mathrm{~mm}$ ), 20 medium aneurysms $(6-15 \mathrm{~mm})$, and 5 large aneurysms $(16-25 \mathrm{~mm})$. There were no giant aneurysms in our series. Thirteen patients $(36.1 \%)$ had additional aneurysms in other locations. Nine patients (25\%) presented with SAH. Of the 9 patients presenting with SAH, the Hunt and Hess scale score was 2 in 4 cases, 3 in 2 cases, and 5 in 3 cases. One of the patients presenting with $\mathrm{SAH}$ was found to have an additional unruptured aneurysm in the contralateral ICA bifurcation. Of the 27 patients with unruptured aneurysms, 3 (11.1\%) were considered symptomatic (a visual field deficit in 1 patient and headache in 2 patients).

Aneurysm Treatment and Immediate Angiographic Outcome. Of the 37 aneurysms, 35 (94.6\%) were initially treated with coiling and $2(5.4 \%)$ were treated in a staged fashion with coiling and flow diversion. Balloon assistance was used in 6 patients undergoing coiling (17.1\%), and stent assistance was not used in any patient. The patients treated with staged coiling and flow diversion had large aneurysms (a ruptured 21-mm aneurysm and an unruptured $23-\mathrm{mm}$ aneurysm). Of the 37 treated aneurysms, 14 (38.8\%) had complete occlusion and 23 (61.2\%) had near-complete occlusion immediately after the procedure. No patients had incomplete occlusion. The mean percentage of occlusion was $80.8 \pm 15 \%$ immediately after the procedure.

\section{Periprocedural Complications}

There were no cases of intraprocedural rupture or perforation and no cases of parent artery or branch occlusion. One of the patients treated with coiling for an unruptured ICA bifurcation aneurysm had a thromboembolic event 10 days after treatment, presenting with a transient neurologic deficit (dysarthria and word-finding difficulty). There were no intra- or periprocedural complications related to the use of the Pipeline Embolization Device (Covidien, Irvine, California). There was no mortality related to the endovascular procedure. As a result, in our institutional series, we had a combined procedure-related morbidity of $2.7 \%$ and no mortality.

\section{Clinical Outcome}

Of the 9 patients presenting with ruptured aneurysms, 2 died in-hospital of complications related to high-grade SAH (Hunt and Hess scale grade 5). Clinical follow-up was available for the 7 patients surviving discharge (mean, 60.6 months; range, 6.8-102 months). The mRS score at last clinical follow-up was zero in 3 patients, 1 in 3 patients, and 2 in 1 patient.

Clinical follow-up was available for 27 of 28 patients with unruptured aneurysms (mean, 44.4 months; range, 2-180 months). The mRS score at last clinical follow-up was zero in 8 patients (26.6\%), 1 in 12 patients (44.5\%), 2 in 4 patients (14.8\%), 3 in 1 patient $(3.7 \%)$, and 6 in 2 patients $(5.9 \%)$. Of the 2 patients with mRS 6, one died of unrelated causes 4 months after treatment and 1 patient died after rupture of a recurrent giant ICA bifurcation aneurysm 30 months after coiling. She had a known recurrence, but given her advanced age and borderline medical condition, we had elected to manage it conservatively.

AJNR Am J Neuroradiol 35:1948-53 Oct 2014 www.ajnr.org 
Table 1: Studies reporting endovascular treatment of ICA terminus aneurysms

\begin{tabular}{lcccccc} 
Authors and Year & $\begin{array}{c}\text { No. of Patients/ } \\
\text { Aneurysms }\end{array}$ & $\begin{array}{c}\text { Male/Female } \\
\text { Ratio }\end{array}$ & $\begin{array}{c}\text { Mean Age (yr) } \\
\text { (Range) }\end{array}$ & $\begin{array}{c}\text { Study } \\
\text { Design }\end{array}$ & $\begin{array}{c}\text { Ruptured Aneurysms } \\
\text { (No.) (\%) }\end{array}$ & $\begin{array}{c}\text { Mean Angiographic } \\
\text { Follow-Up (mo) }\end{array}$ \\
\hline Geyik et al, 2007 (14 $^{16}$ & $18 / 18$ & NR & $31.9(21-65)$ & $\mathrm{R}$ & $\mathrm{NR}$ & 28 \\
Uemura et al, 2008 & $17 / 17$ & $9: 8$ & $47(24-77)$ & $\mathrm{R}$ & $13(76.5 \%)$ & 12 \\
van Rooij et al, 2008 & $46 / 50$ & $13: 33$ & $49.3(23-76)$ & $\mathrm{R}$ & $26(52 \%)$ & 16 \\
Oishi et al, 2013 & $25 / 25$ & $11: 14$ & $60.9(45-77)$ & $\mathrm{P}$ & $2(8 \%)$ & 24 \\
Zhou et al, 2013 $^{15}$ & $16 / 16$ & $9: 7$ & $50.6(34-66)$ & $\mathrm{R}$ & $8(50 \%)$ & 12 \\
Current study & $36 / 37$ & $14: 22$ & $50.7(22-80)$ & $\mathrm{R}$ & $9(24.3 \%)$ & 40 \\
\hline
\end{tabular}

Note:-NR indicates not reported; R, retrospective; $P$, prospective.

\section{Long-Term Angiographic Outcome}

Angiographic follow-up was available for $88 \%$ of eligible patients (30/34). The average interval between initial treatment and last imaging follow-up was 39.5 months (range, 2-156.8 months). Of the 4 patients without available imaging follow-up, 2 were recently treated and had no indication of repeat imaging at the time of data collection and 2 had follow-up angiograms obtained at other institutions, which were not available for review. At last imaging follow-up, 14 of 30 patients ( $46.7 \%$ ) had complete occlusion, 9 (30.0\%) had near-complete occlusion, and 7 (23.3\%) had incomplete occlusion. The mean percentage of occlusion was $85 \pm 15.6 \%$ at last angiographic follow-up.

\section{Recurrence and Retreatment}

Overall, 34.3\% (12/35) of aneurysms recurred after a mean of 13.3 months (range, 2-41 months). One recurrent aneurysm presented as rebleeding 2 months after the initial SAH and coiling; the remaining recurrent aneurysms were diagnosed during routine angiographic follow-up. By size, $41.7 \%$ of the small aneurysms recurred (5/12), as opposed to $25 \%$ of the medium aneurysms (5/20), and 2 of 3 large aneurysms. Nine aneurysms (25.7\%) underwent retreatment: 6 with coiling, 2 with the Pipeline Embolization Device, and 1 with surgical clipping. Of the 3 patients with recurrent aneurysms that did not undergo retreatment, 2 aneurysms were not amenable to coiling and were thought to have a low risk of rupture and 1 patient was managed conservatively due to advanced age and a borderline medical condition. The 2 patients treated with the Pipeline Embolization Device included 1 with a 12-mm aneurysm initially treated with coiling and found to have recurred 28 months later and 1 with a large (23-mm) aneurysm initially treated with staged coiling and flow diversion found to have recurred during the 6-month angiographic follow-up. In this patient, further growth of the aneurysm was related to proximal delayed migration of the Pipeline Device. Retreatment with 2 additional telescoping Pipelines was complicated by a nondisabling stroke causing transient word-finding difficulties 1 week after treatment. The last angiographic follow-up on this patient 36 months after initial treatment showed complete obliteration. Similarly, the other patient initially treated with staged coiling and flow diversion showed complete obliteration of the aneurysm during the 12-month angiographic follow-up.

\section{Systematic Review and Meta-Analysis}

Search Results. The search criteria led to 171 citations. After screening the titles and abstracts, we identified 15 articles about treatment of ICA bifurcation aneurysms. Of these 15 articles, 5 were case reports and were excluded. Three studies included pa- tients treated with surgery only and were excluded as well. Three large studies of endovascular treatment in various locations included patients with ICA bifurcation aneurysms, but only 1 reported disaggregated data and met our inclusion criteria. ${ }^{14}$ Four series included exclusively patients with ICA bifurcation aneurysms undergoing endovascular treatment and were articles included in our analysis. ${ }^{4,15-17}$ Thus, our search results yielded 5 published series on endovascular treatment of ICA bifurcation aneurysms.

\section{Studies}

Including the institutional series presented in this article, 6 studies encompassing 158 patients with 163 aneurysms were included in this meta-analysis (Table 1). Five studies with 145 aneurysms provided data regarding aneurysm rupture status; among them, 87 $(60 \%)$ corresponded to unruptured aneurysms. All studies provided data on technical outcome, periprocedural complications, procedure-related morbidity and mortality, immediate and longterm angiographic outcomes, and clinical outcome.

\section{Angiographic Outcomes}

The rate of complete or near-complete occlusion immediately after the endovascular procedure was $88.0 \%$ (95\% CI, $68.0 \%-$ $96.0 \%)$ for all aneurysms. The occlusion rate for unruptured aneurysms was $84.0 \%$ (95\% CI, 36.0\%-98.0\%), and for ruptured aneurysms, it was $69.0 \%$ (95\% CI, 4.0\%-99.0\%). Long-term complete/near-complete occlusion rates were $82.0 \%$ (95\% CI, $73.0 \%-88.0 \%$ ) for all aneurysms. These data are summarized in Table 2.

\section{Procedure-Related Complications}

Overall, perioperative strokes resulting in morbidity or mortality occurred in $3.0 \%$ (95\% CI, 1.0\%-7.0\%) of patients. Intraoperative rupture occurred in $3.0 \%$ (95\% CI, $1.0 \%-7.0 \%$ ) of patients. Procedure-related morbidity occurred in $4.0 \%$ (95\% CI, $2.0 \%-$ $14.0 \%)$, and procedure-related mortality occurred in $3.0 \%$ (95\% CI, $1.0 \%-8.0 \%)$ of all patients. Procedure-related morbidity occurred in $6.0 \%$ (95\% CI, $2.0 \%-14.0 \%)$ of patients with unruptured aneurysms and $9.0 \%$ (95\% CI, 2.0\%-36.0\%) of patients with ruptured aneurysms. Procedure-related mortality occurred in $4.0 \%$ (95\% CI, $1.0 \%-11.0 \%)$ of patients with unruptured aneurysms and $6.0 \%$ (95\% CI, $2.0 \%-16.0 \%)$ of patients with ruptured aneurysms. These data are summarized in Table 2.

\section{Long-Term Outcome}

The mean duration of clinical follow-up among the included studies was $31.2 \pm 9.7$ months. The overall retreatment rate was 


\begin{tabular}{|c|c|c|c|c|c|c|c|c|c|}
\hline & \multicolumn{3}{|c|}{ All Patients } & \multicolumn{3}{|c|}{ Unruptured Aneurysm } & \multicolumn{3}{|c|}{ Ruptured Aneurysm } \\
\hline & $\begin{array}{l}\text { Event Rate } \\
(95 \% \mathrm{Cl})\end{array}$ & $1^{2}$ & $\begin{array}{l}\text { No. of } \\
\text { Studies }\end{array}$ & $\begin{array}{l}\text { Event Rate } \\
(95 \% \mathrm{Cl})\end{array}$ & $1^{2}$ & $\begin{array}{l}\text { No. of } \\
\text { Studies }\end{array}$ & $\begin{array}{l}\text { Event Rate } \\
(95 \% \mathrm{Cl})\end{array}$ & $I^{2}$ & $\begin{array}{l}\text { No. of } \\
\text { Studies }\end{array}$ \\
\hline \multicolumn{10}{|l|}{ Angiographic outcome } \\
\hline $\begin{array}{l}\text { Immediate complete/near-complete } \\
\text { occlusion }\end{array}$ & $88.0(68.0-96.0)$ & 80 & 6 & $84.0(36.0-98.0)$ & 79 & 2 & $69.0(4.0-99.0)$ & 84 & 4 \\
\hline $\begin{array}{l}\text { Complete/near-complete occlusion } \\
\text { at last follow-up }\end{array}$ & $82.0(73.0-88.0)$ & 0 & 5 & $82.0(66.0-92.0)$ & NA & 2 & $67.0(40.0-85.0)$ & NA & 2 \\
\hline \multicolumn{10}{|l|}{ Perioperative clinical outcomes } \\
\hline Procedure-related morbidity & $4.0(2.0-9.0)$ & 0 & 6 & $6.0(2.0-14.0)$ & 0 & 5 & $9.0(2.0-36.0)$ & 50 & 5 \\
\hline Procedure-related mortality & $3.0(1.0-8.0)$ & 0 & 6 & $4.0(1.0-11.0)$ & 0 & 5 & $6.0(2.0-16.0)$ & 0 & 5 \\
\hline Intraoperative rupture & $3.0(1.0-7.0)$ & 0 & 6 & $3.0(1.0-11.0)$ & 0 & 5 & $7.0(2.0-19.0)$ & 0 & 5 \\
\hline $\begin{array}{l}\text { Perioperative stroke resulting in } \\
\text { morbidity or mortality }\end{array}$ & $3.0(1.0-7.0)$ & 0 & 6 & $3.0(1.0-11.0)$ & 0 & 5 & $7.0(3.0-17.0)$ & 0 & 5 \\
\hline \multicolumn{10}{|l|}{ Long-term clinical outcomes } \\
\hline Retreatment & $14.0(8.0-25.0)$ & 37 & 6 & $14.0(8.0-25.0)$ & 37 & 6 & $22.0(7.0-51.0)$ & 45 & 4 \\
\hline Good neurologic outcome long-term & $93.0(86.0-97.0)$ & 0 & 4 & $91.0(82.0-96.0)$ & 0 & 4 & $93.0(79.0-98.0)$ & 0 & 4 \\
\hline
\end{tabular}

Note:-NA indicates not applicable.

Table 3: SAH patient-specific outcomes

\begin{tabular}{lcrc}
\hline & $\begin{array}{c}\text { Event Rate } \\
(95 \% \mathrm{CI})\end{array}$ & $\mathbf{I}^{\mathbf{2}}$ & $\begin{array}{c}\text { No. of } \\
\text { Studies }\end{array}$ \\
\hline Mortality from SAH & $14.0(7.0-26.0)$ & 0 & 5 \\
Vasospasm & $16.0(4.0-46.0)$ & 40 & 4 \\
Rerupture after coiling & $6.0(2.0-17.0)$ & 0 & 5 \\
\hline
\end{tabular}

14.0\% (95\% CI, 8.0\%-25.0\%). The retreatment rate for unruptured aneurysms was $14.0 \%$ (95\% CI, 8.0\%-25.0\%), and for ruptured aneurysms, it was $22.0 \%$ (95\% CI, 7.0\%-51.0\%). Overall, good long-term neurologic outcome was seen in $93.0 \%$ (95\% CI, $86.0 \%-97.0 \%)$ of patients. Among patients with available longterm clinical follow-up, those with unruptured aneurysms had a good neurologic outcome rate of $91.0 \%$ (95\% CI, 82.0\%-96.0\%), and those with ruptured aneurysms had a good neurologic outcome rate of $93.0 \%$ (95\% CI, 79.0\%-98.0\%). These data are summarized in Table 2.

\section{SAH-Specific Outcomes}

Among patients included in the meta-analysis, mortality from SAH occurred in $14.0 \%$ (95\% CI, 7.0-26.0\%) of patients. Vasospasm occurred in $16.0 \%$ (95\% CI, 4.0\%-46.0\%). Rerupture after coiling occurred in $6.0 \%$ (95\% CI, $2.0 \%-17.0 \%)$. These data are summarized in Table 3.

\section{Heterogeneity and Publication Bias}

Heterogeneity was low for all outcomes except immediate complete/near-complete occlusion $\left(\mathrm{I}^{2}=80 \%\right)$. Therefore, variation in this event rate was noted across the available studies, whereas results were consistent for all the remaining outcomes. We were unable to determine the presence of publication bias due to the small number of included studies and the noncomparative nature of the available data.

\section{DISCUSSION}

Reporting our institutional experience with the endovascular treatment of ICA bifurcation aneurysms, we contribute to the literature with a relatively large contemporary series with the longest clinical and angiographic follow-up available to date, to our knowledge. Additionally, by combining our results with 5 previ- ous studies, we are able to provide representative data and draw accurate conclusions regarding the safety and efficacy of endovascular treatment of ICA bifurcation aneurysms.

Most ICA terminus aneurysms in our meta-analysis were successfully embolized with a rate of complete or near-complete occlusion of $88 \%$ in the immediate postoperative setting. A potential limitation of endovascular treatment for aneurysms at bifurcation points is the possibility of coil compaction and subsequent aneurysm recurrence. Our data indicate that retreatment was necessary in $14 \%$ of patients undergoing endovascular treatment for ICA bifurcation aneurysms. However, complete or nearcomplete occlusion was seen in $82 \%$ of patients after long-term imaging follow-up.

Periprocedural complications following endovascular treatment of ICA terminus aneurysms are not negligible. Aneurysms at this location are at a high risk of ischemic stroke in the territory of the ipsilateral MCA (either from distal emboli during the procedure or clot formation at the level of the neck with impairment of distal MCA flow), a potential source of serious morbidity and mortality. ${ }^{4,16,17}$ In our meta-analysis, perioperative morbidity rates were approximately $4 \%$ and mortality rates were $3 \%$. Perioperative stroke was a major contributor to morbidity and mortality, occurring in approximately $3 \%$ of patients. Although good long-term neurologic outcome was achieved in $>90 \%$ of patients regardless of aneurysm rupture status, the periprocedural complication rate reported is not trivial. Particularly worrisome is the procedure-related mortality of $4 \%$ for unruptured and $6 \%$ for ruptured ICA bifurcation aneurysms.

These findings stress the importance of proper patient selection because these aneurysms are often adequately and effectively treatable with surgical clip ligation. The high retreatment rate observed in our meta-analysis and in our own series is similar to that reported for aneurysms located in other bifurcation points. The retreatment rate reported for basilar bifurcation aneurysms ranges between $15 \%$ and $27.8 \%,{ }^{18-20}$ and for MCA bifurcation aneurysms, it has been reported in up to $7.6 \%$ of patients. ${ }^{21}$ The increased hemodynamic stress at the level of arterial bifurcations has been linked to the development of aneurysms $s^{5,22}$ and certainly has a role in the recurrence seen after endovascular treatment. Most interesting, some aneurysms were found to have recurred 
even after stable follow-up imaging 6 or 12 months after initial treatment. This finding underscores the need for close angiographic follow-up in this patient population as stressed by other authors. $^{23}$

Given that aneurysms located at the ICA bifurcation are relatively uncommon, the series included in our analysis had a small number of patients. Additionally, there was a wide range of angiographic outcomes among the different studies. By pooling the results of all available reports on endovascular treatment of ICA bifurcation aneurysms, our meta-analysis provides a more precise estimate of the safety and efficacy of this treatment technique for this particular group of aneurysms.

The surgical treatment of ICA bifurcation aneurysms is particularly challenging, due to their location at the highest point of the ICA and the presence of multiple perforators at this level that may be adherent to the back side of the aneurysm. ${ }^{3}$ Results of endovascular treatment herein reported compare favorably with those observed after surgical treatment. Gupta el at ${ }^{10}$ reported their experience with 89 patients with ruptured ICA bifurcation aneurysms and showed a good outcome in $68.6 \%$. Although all of their patients presented with ruptured aneurysms, even patients with low-grade SAH (Hunt and Hess scale grades I and II) had a mortality rate of $6.3 \%$, mainly due to intraoperative rupture and thromboembolic complications. ${ }^{10}$ Miyazawa et $\mathrm{al}^{2}$ reported a good outcome in $58.8 \%$ of patients with ruptured aneurysms and in all patients with unruptured aneurysms. In our study, good neurologic outcome was seen in $>90 \%$ of patients, and $>80 \%$ of patients had complete or near-complete occlusion of their aneurysms. These findings suggest that endovascular treatment could be considered a first-line treatment for ICA terminus aneurysms.

Treatment in a staged fashion by using coiling and flow diversion was used in 2 large aneurysms from our series. To our knowledge, this technique has not been reported previously for the treatment of ICA bifurcation aneurysms, and it proved effective in both cases. The safety and efficacy of flow diversion are welldocumented for the treatment of sidewall aneurysms located in the proximal ICA. ${ }^{24,25}$ However, this new technology has been increasingly used to treat aneurysms at other locations. A recent study reported $25 \mathrm{MCA}$ bifurcation aneurysms treated by the Pipeline Embolization Device, showing complete occlusion in $84 \%$ of cases at last angiographic follow-up. ${ }^{26}$ In our case, both patients with ICA bifurcation aneurysms and treated with staged coiling and flow diversion had complex and large lesions that were thought to have a high risk of recurrence if treated by conventional modalities. We consider that selected cases of aneurysms located at the ICA bifurcation represent a potential application of flow diverters. However, further evidence is required to confirm this assumption.

\section{Limitations}

Our study has methodologic limitations. Measurement of aneurysm occlusion by using both the 3-point scale of Raymond et al, ${ }^{11}$ and the percentage occlusion scale can be subjective and subject to both inter- and intraobserver variability. For the meta-analysis, clinical and angiographic follow-up times were variable, the average ranging from 12 to 40 months. Thus, data on clinical and angiographic outcomes are limited by the heterogeneity in follow- up. In addition, many of the series analyzed and included in our analysis were cases collected for several years, and it is possible that complication rates have improved as a result of increased operator experience and skill and improved devices and technology. Using the Grading of Recommendations, Assessment, Development and Evaluation framework, we found that the quality of evidence (confidence in estimates) is limited because of imprecision, heterogeneity (only for the outcome of immediate complete/near-complete occlusion), and methodologic limitations of the studies included. ${ }^{27-29}$

Nevertheless, this meta-analysis provides useful data to share with patients and families when assessing the risks of endovascular treatment of ICA bifurcation aneurysms. Our study also provides a framework on which to base the treatment decision-making for these uncommon lesions and represents a benchmark against which future studies in this patient group can be compared. With 158 patients and 163 aneurysms, this study represents the largest study to date on the endovascular treatment of ICA terminus aneurysms.

\section{CONCLUSIONS}

Endovascular treatment of ICA bifurcation aneurysms is feasible and results in immediate complete or near-complete occlusion in $88 \%$ of patients. However, non-negligible complications can occur, and the procedure-related morbidity and mortality are $4 \%$ and $3 \%$, respectively. ICA bifurcation aneurysms are subject to increased hemodynamic stress, which explains their tendency to recur $(34.3 \%$ in our own series), requiring a retreatment rate of $14 \%$. With close angiographic follow-up and adequate treatment of recurrences, most patients achieve a good neurologic outcome.

Disclosures: Giuseppe Lanzino-UNRELATED: Consultancy: ev3/Covidien*; Consulting fee or honorarium: Codman/Johnson \& Johnson. *Money paid to the institution.

\section{REFERENCES}

1. Sakamoto S, Ohba S, Shibukawa M, et al. Characteristics of aneurysms of the internal carotid artery bifurcation. Acta Neurochir (Wien) 2006;148:139-43, discussion 143

2. Miyazawa N, Nukui H, Horikoshi T, et al. Surgical management of aneurysms of the bifurcation of the internal carotid artery. Clin Neurol Neurosurg 2002;104:103-14

3. Lehecka M, Dashti R, Romani R, et al. Microneurosurgical management of internal carotid artery bifurcation aneurysms. Surg Neurol 2009;71:649-67

4. van Rooij WJ, Sluzewski M, Beute GN. Internal carotid bifurcation aneurysms: frequency, angiographic anatomy and results of coiling in 50 aneurysms. Neuroradiology 2008;50:583-87

5. Ingebrigtsen T, Morgan MK, Faulder K, et al. Bifurcation geometry and the presence of cerebral artery aneurysms. J Neurosurg 2004;101:108-13

6. Perria L, Rivano C, Rossi GF, et al. Aneurysms of the bifurcation of the internal carotid artery. Acta Neurochir (Wien) 1968;19:51-68

7. Sengupta RP, Lassman LP, de Moraes AA, et al. Treatment of internal carotid bifurcation aneurysms by direct surgery. J Neurosurg 1975;43:343-51

8. Yaşargil MG, Boehm WB, Ho RE. Microsurgical treatment of cerebral aneurysms at the bifurcation of the internal carotid artery. Acta Neurochir (Wien) 1978;41:61-72

9. Da Pian R, Pasqualin A, Scienza R. Direct microsurgical approach to aneurysms of the internal carotid bifurcation. Surg Neurol 1980;13:27-37 
10. Gupta SK, Khosla VK, Chhabra R, et al. Internal carotid artery bifurcation aneurysms: surgical experience. Neurol Med Chir (Tokyo) 2007;47:153-57, discussion 157-58

11. Raymond J, Guilbert F, Weill A, et al. Long-term angiographic recurrences after selective endovascular treatment of aneurysms with detachable coils. Stroke 2003;34:1398-403

12. DerSimonian R, Laird N. Meta-analysis in clinical trials. Control Clin Trials 1986;7:177-88

13. Higgins JP, Thompson SG, Deeks JJ, et al. Measuring inconsistency in meta-analyses. BMJ 2003;327:557-60

14. Geyik S, Yavuz K, Cekirge S, et al. Endovascular treatment of basilar and ICA termination aneurysms: effects of the use of HydroCoils on treatment stability in a subgroup of patients prone to a higher recurrence rate. Neuroradiology 2007;49:1015-21

15. Zhou Y, Yang PF, Hong B, et al. Stent placement for the treatment of complex internal carotid bifurcation aneurysms: a review of 16 cases. Turk Neurosurg 2013;23:232-40

16. Uemura A, Musacchio M, Cardoso M, et al. Internal carotid bifurcation aneurysms: anatomical features and outcome of endovascular treatment. Neuroradiol J 2008;21:574-78

17. Oishi H, Yamamoto M, Nonaka S, et al. Endovascular therapy of internal carotid artery bifurcation aneurysms. J Neurointerv Surg 2013;5:400-04

18. Chalouhi N, Jabbour P, Gonzalez LF, et al. Safety and efficacy of endovascular treatment of basilar tip aneurysms by coiling with and without stent assistance: a review of 235 cases. Neurosurgery 2012;71:785-94

19. Peluso JP, van Rooij WJ, Sluzewski M, et al. Coiling of basilar tip aneurysms: results in 154 consecutive patients with emphasis on recurrent haemorrhage and re-treatment during mid- and longterm follow-up. J Neurol Neurosurg Psychiatry 2008;79:706-11

20. Henkes H, Fischer S, Mariushi W, et al. Angiographic and clinical results in $\mathbf{3 1 6}$ coil-treated basilar artery bifurcation aneurysms. J Neurosurg 2005;103:990-99

21. Gory B, Rouchaud A, Saleme S, et al. Endovascular treatment of middle cerebral artery aneurysms for 120 nonselected patients: a prospective cohort study. AJNR Am J Neuroradiol 2014;35:715-20

22. Meng H, Wang Z, Hoi Y, et al. Complex hemodynamics at the apex of an arterial bifurcation induces vascular remodeling resembling cerebral aneurysm initiation. Stroke 2007;38:1924-31

23. Ferns SP, Sprengers ME, van Rooij WJ, et al. Late reopening of adequately coiled intracranial aneurysms: frequency and risk factors in 400 patients with 440 aneurysms. Stroke 2011;42:1331-37

24. Brinjikji W, Murad MH, Lanzino G, et al. Endovascular treatment of intracranial aneurysms with flow diverters: a meta-analysis. Stroke 2013;44:442-47

25. D’Urso PI, Lanzino G, Cloft HJ, et al. Flow diversion for intracranial aneurysms: a review. Stroke 2011;42:2363-68

26. Yavuz K, Geyik S, Saatci I, et al. Endovascular treatment of middle cerebral artery aneurysms with flow modification with the use of the Pipeline embolization device. AJNR Am J Neuroradiol 2014;35:529-35

27. Balshem $H$, Helfand $M$, Schunemann HJ, et al. GRADE guidelines: 3 . Rating the quality of evidence. J Clin Epidemiol 2011;64:401-06

28. Guyatt GH, Oxman AD, Kunz R, et al. GRADE guidelines: 6. Rating the quality of evidence-imprecision. J Clin Epidemiol 2011;64:1283-93

29. Murad MH, Swiglo BA, Sidawy AN, et al. Methodology for clinical practice guidelines for the management of arteriovenous access. $J$ Vasc Surg 2008;48:26S-30S 